\title{
First-principles calculations of Pd-terminated symmetrical armchair graphene nanoribbons
}

\author{
A.F. Kuloglu ${ }^{\text {a }}$, B. Sarikavak-Lisesivdin ${ }^{\text {a }}$, S.B. Lisesivdin ${ }^{\text {a,* }}$, E. Ozbay ${ }^{\text {b,c }}$ \\ ${ }^{a}$ Gazi University, Faculty of Science, Department of Physics, 06500 Teknikokullar, Ankara, Turkey \\ ${ }^{\mathrm{b}}$ Bilkent University, Nanotechnology Research Center, 06800 Bilkent, Turkey \\ ${ }^{\mathrm{c}}$ Bilkent University, Department of Physics, Department of Electrical and Electronics Engineering, 06800 Bilkent, Turkey
}

\section{A R T I C L E I N F O}

\section{Article history:}

Received 24 July 2012

Received in revised form 17 October 2012

Accepted 19 October 2012

Available online 15 November 2012

\section{Keywords:}

Palladium

Termination

Passivation

Graphene

GNR

Nanoribbon

\begin{abstract}
A B S T R A C T
The effects of Palladium (Pd) termination on the electronic properties of armchair graphene nanoribbons (AGNRs) were calculated by using $a b$ initio calculations. After a geometric optimization process, the electronic band structures, density of states, and binding energies of AGNRs with $N_{a}=5-15$ were calculated. Pd-termination was found to significantly influence the electronic properties of AGNRs. In DOS, many Q0D and Q1D type states were observed. Binding energy (BE) for single-side or both-side Pd-terminated structures represents characteristic drops with the increasing GNR width. With the increasing GNR width, the BEs of these structures become similar to hydrogenated structures. Because of the GNR width, dependent $\mathrm{BE}$ also gave information on the possible stiffness information, in which all of this information can be used in studies where controlled binding to graphene is required.
\end{abstract}

(c) 2012 Elsevier B.V. All rights reserved.

\section{Introduction}

The two-dimensional (2D) crystals of $\mathrm{sp}^{2}$ bonded a carbon (C) atom, which is called graphene, shows different structural and electronic properties when compared with other carbon allotropes. Until 2004, graphene was believed to be thermodynamically unstable under ambient conditions. After Novoselov et al. showed the availability of massless 2D Dirac fermions in high quality free-standing graphene sheets [1], a significant number of researchers started to work on this topic experimentally and theoretically [2-4]. The high number of studies resulted in a more successfully controlled quasi-one-dimensional (Q1D) form, which are the so-called graphene nanoribbons (GNRs) [5].

It is widely known that the electronic, magnetic, and structural properties of GNRs are highly dependent on the chirality and size of the GNRs [6-8]. Armchair-edged GNRs show semiconductor behavior, and all zigzag-edged GNRs are known to be metallic [9].

The scanning tunneling microscopy (STM) measurements of hydrogen $(\mathrm{H})$-terminated the graphite present high-density edge states near the Fermi level [10]. This observation shows the importance of the edge states for electrical properties of GNRs. From a chemistry point-of-view, zigzag and armchair sites in a graphene sheet behave very differently as carbene-like and carbyne-like, respectively [11]. Therefore, investigating edge states formed by

\footnotetext{
* Corresponding author. Fax: +90 3122122279.

E-mail address: sblisesivdin@gmail.com (S.B. Lisesivdin).
}

H-terminated or different terminated zigzag, armchair GNRs, or GNRs with chirality has gained in importance [12].

With the increasing number of studies, graphene synthesis on the non-carbide substrates by epitaxial methods is becoming one of the active subjects of study. Recently, the graphene growth results on transition metals, such as Ru [13], Ir [14], Pt [15], Cu [16], Ni [17], and Pd [18-20], have been reported.

The experimental attempts for the graphene growth on $\operatorname{Pd}(111)$ by chemical vapor deposition (CVD) demonstrated the possibility of graphene formation [18,19]. A current study reported a uniform, large-area, single-layer epitaxial growth of graphene on a carbondoped $\operatorname{Pd}(111)$ substrate by surface segregation [20]. This approach provides for the controlled fabrication of single-layer graphene and, therefore, an understanding of the interaction of graphene and Pd is of great importance. Theoretical studies about this subject are likely to be concentrated on Pd-decorated [21,22] or Pd-cluster adsorbed [23,24] graphene structures because of the possible $\mathrm{H}$-storage capability.

In this study, the electronic properties and binding energies of graphene nanoribbons with symmetrical armchair edges were investigated, depending on the ribbon width and edge termination by using the Density Functional Theory (DFT).

\section{Computational method}

The calculations were performed by using Atomistix Toolkit-Visual NanoLab (ATK-VNL) [25-27]. For the $x$ - and $y$-axes vacuum 
confinement and for the $z$-axis, periodic boundary conditions are accepted. For the vacuum confinement region, the interspace between two periodic slabs was kept enough to avoid the interaction. Therefore, our structure can be accepted as single layer graphene. All atoms were fully relaxed and maximum strains on atoms were accepted to be less than $0.005 \mathrm{eV} / \mathrm{A}$. As an exchange correlation function, the local density approximation (LDA) was used in the form of Perdew and Zunger [28] with an energy cut-off value of 100 Ry. The main reason for choosing LDA over generalized gradient approximation (GGA) in this study can be attributed to the fact that underestimation of interactions of graphene with atoms and molecules in GGA [29]. Accordingly, the binding energy and the amount of charge transfer of molecule adsorption on graphene and nanotubes by GGA are also smaller than the prediction from LDA [29]. In spite of giving better results for interactions, previous theoretical studies have shown that the LDA somehow underestimates the band gap for quasi one-dimensional semiconducting systems such as armchair GNRs [30]. Although LDA gives smaller bandgaps, in this work, we mostly focus on the variations of binding energies of Pd-terminated systems, and these changes with respect to the nanoribbon widths are quantitatively acceptable. The Brilliouin zone integration was used with a regular MonkhorstPack $k$-point grid of $1 \times 1 \times 100$ [31]. Symmetrical armchair GNR structures with $N_{a}$ dimer lines are investigated in this study. These structures are referred to as $N_{a}$-AGNR. For the symmetrical AGNRs that are calculated, $N_{a}$ is taken as 5-15 (odd numbered) with both side $\mathrm{H}$-terminated $(\mathrm{H}-\mathrm{C}-\mathrm{H})$, one side Pd-terminated, and one side $\mathrm{H}$-terminated ( $\mathrm{H}-\mathrm{C}-\mathrm{Pd})$, both-side Pd-terminated (Pd-C-Pd) in addition to bare structures. In Fig. 1, supercells of (a) bare, (b) $\mathrm{H}-\mathrm{C}-\mathrm{H}$, (c) H-C-Pd and (d) Pd-C-Pd 11-AGNR structures are shown as an example.

\section{Results and discussion}

Pd atoms are not naturally magnetic because of the closed shell configuration of the $4 \mathrm{~d}$ orbital $\left(4 \mathrm{~d}^{10}\right)$. In the calculations, the investigated AGNRs with or without Pd atoms are found to have the same degenerate results for spin included calculations, which means our cases are non-magnetic. With the changing $N_{a}$, the investigated structures show similar trends. Therefore, we prefer to present the results of 11-AGNRs for simplicity.

Band structures of bare, $\mathrm{H}-\mathrm{C}-\mathrm{H}, \mathrm{H}-\mathrm{C}-\mathrm{Pd}$, and $\mathrm{Pd}-\mathrm{C}-\mathrm{Pd}$ cases are calculated for investigated symmetrical AGNRs with $N_{a}=5-15$. The dangling bonds of bare structures result in a decrease in the lattice parameters and show a semiconductor behavior as shown for bare 11-AGNR in Fig. 2a [32]. In Fig. 2b, the band structure of the $\mathrm{H}-\mathrm{C}-\mathrm{H}$ structure is shown. It shows a semiconductor behavior with a direct band gap, which is highly known phenomena [33]. The H-CPd structure, which is shown in Fig. 2c, has a metallic behavior. Pd has a filled d shell, and it binds strongly to the graphene, implying covalent bonding. In Fig. 2d, the band structure of the Pd-C-Pd structure is shown. The band structure is mostly similar to $\mathrm{H}-\mathrm{C}-\mathrm{Pd}$ with a degeneracy that is a conclusion of a symmetrical nature of the terminations. In addition, the number of valence bands is observed to be increased.

In Fig. 3a-d, the density of states (DOS) of these structures is shown in the same order. The DOS of the bare and $\mathrm{H}-\mathrm{C}-\mathrm{H}$ structures, which are shown in Fig. 3a and b, respectively, are found to be symmetric well-known one-dimensional (1D) DOS behavior with changing bandgaps $0.3-1.5 \mathrm{eV}$. These values can be accepted as underestimated with the usage of LDA in our calculations. Bare structures have both indirect and direct bandgaps, but $\mathrm{H}-\mathrm{C}-\mathrm{H}$ structures only have direct bandgaps as is known in the literature

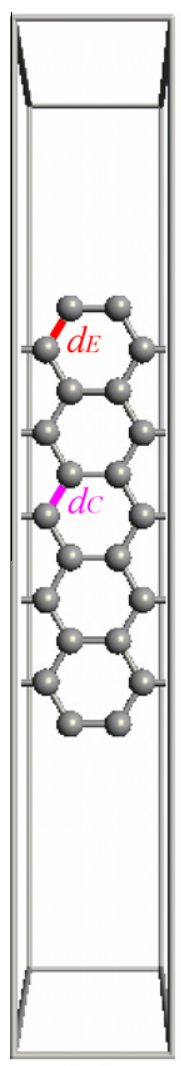

(a)

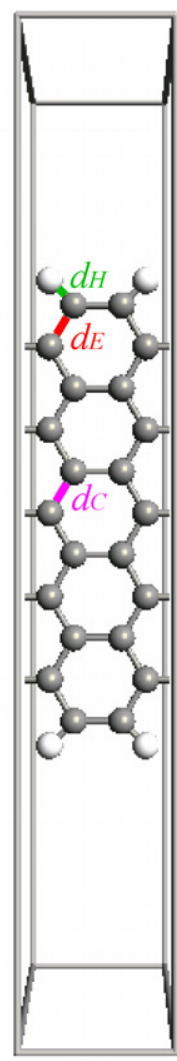

(b)

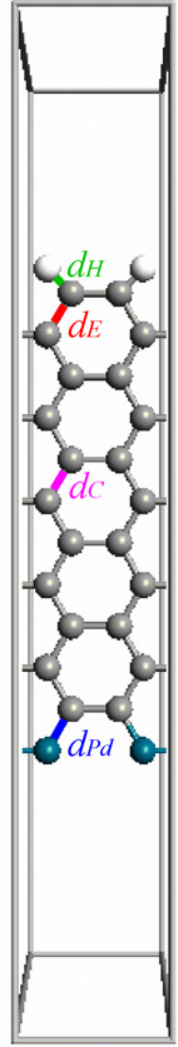

(c)

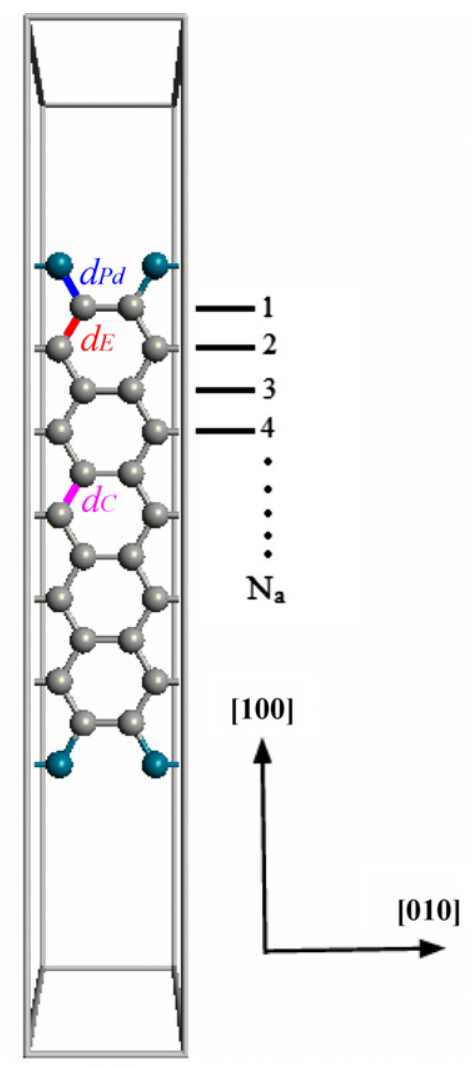

(d)

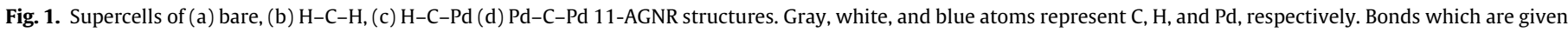
in Table 2 are also shown. (For interpretation of the references to color in this figure legend, the reader is referred to the web version of this article.) 
(a)

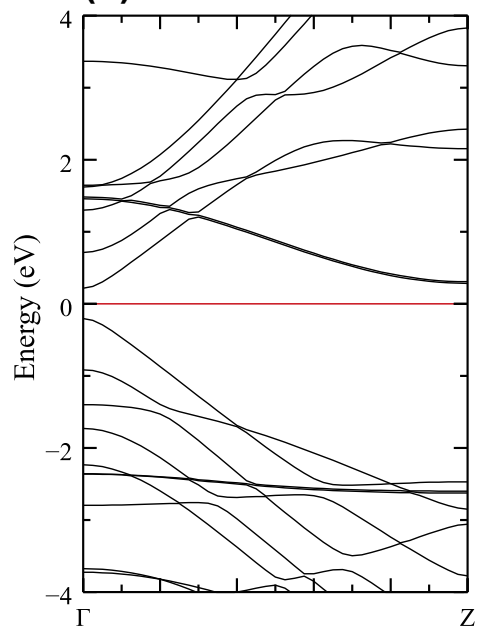

(c)

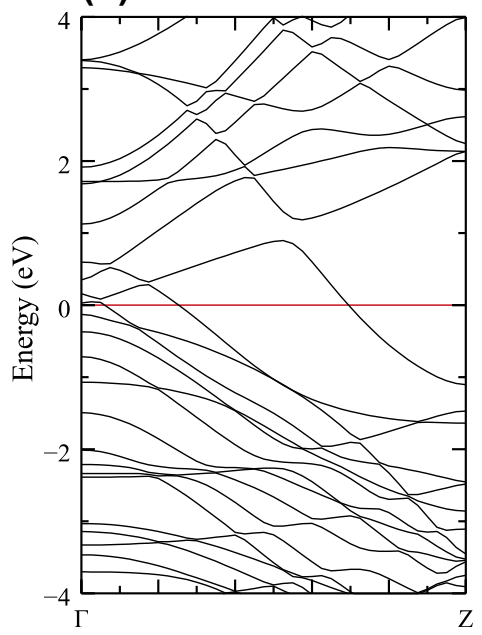

(b)

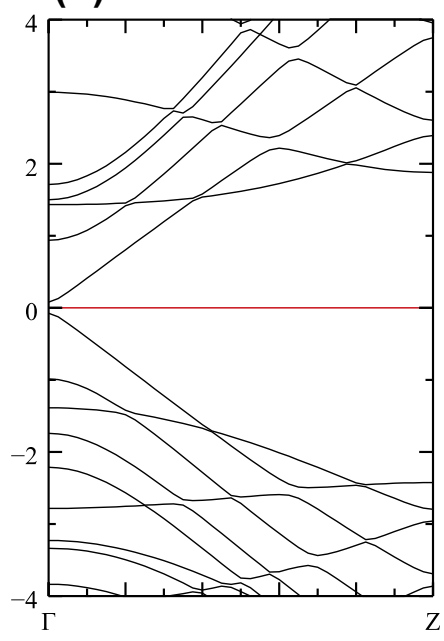

(d)

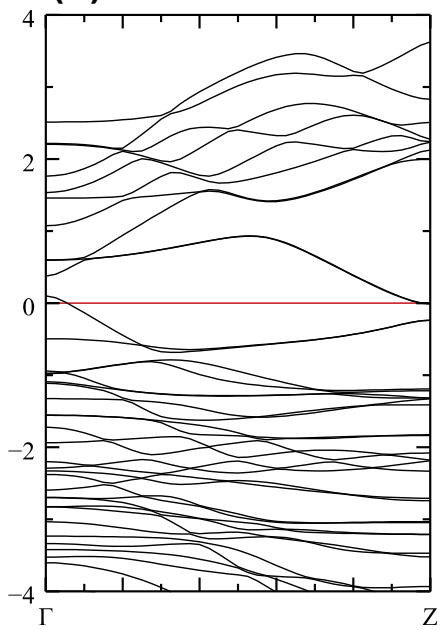

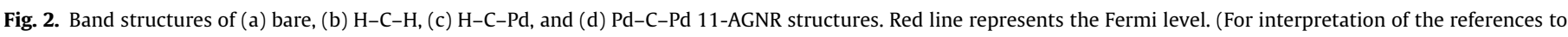
color in this figure legend, the reader is referred to the web version of this article.)

[33,34]. The H-C-Pd and Pd-C-Pd structures do not have band gaps due to the hybridization of Pd and graphene orbitals, which produce a strong enhancement of the density of states near the Fermi surface. In the DOS data of $\mathrm{H}-\mathrm{C}-\mathrm{Pd}$ and $\mathrm{Pd}-\mathrm{C}-\mathrm{Pd}$, many local quasi-one dimensional (Q1D) and quasi-zero dimensional (Q0D) behaviors are observed as shown in Fig. $3 c$ and $d$, respectively. The presence of these Q1D and Q0D states in the investigated structures stand for existence of mixed 2D-1D and 1D-0D behaviors, respectively [35]. For these structures, with the increasing Pd density, a highly localized Pd 4d shell may result in lower dimensional peaks in the DOS spectra.

For the investigated structures with $N_{a}=5-15$, binding energies (BE) are calculated and shown in Fig. 4. For the bare structure and other configurations with different terminations, BEs are calculated as:

$E_{B}=\frac{E_{T, \text { Bare }}}{n}-E_{C} \quad$ (for bare)

and

$E_{B}=E_{T}-E_{T, B a r e}-n E_{H}-m E_{P d} \quad$ (for other configurations)

respectively. Here, $E_{B}, E_{T}, E_{T, B a r e}, E_{C}, E_{H}, E_{P d}, n$ and $m$ are the $\mathrm{BE}$, total energy of the investigated configuration, total energy of bare AGNR, total energy of isolated carbon, hydrogen and palladium atoms,
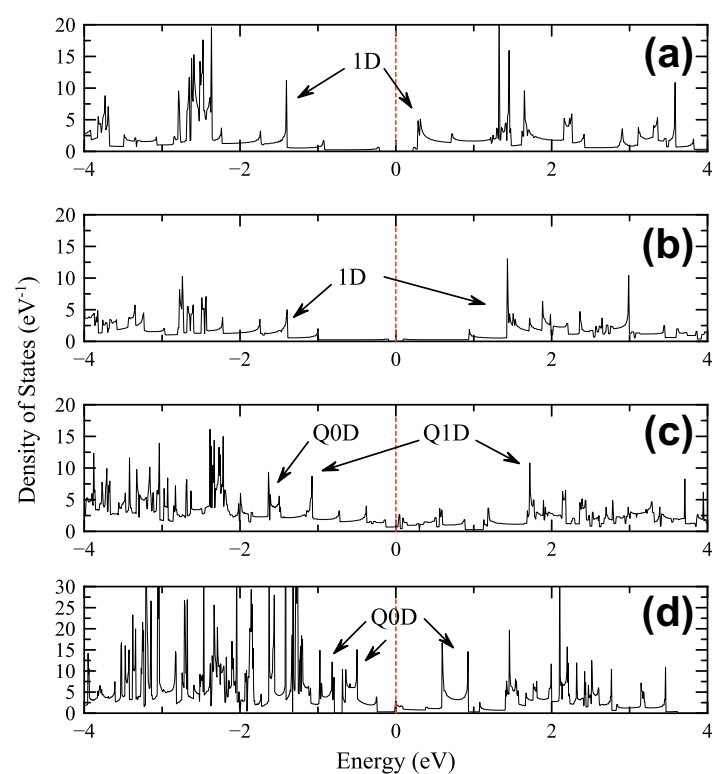

Fig. 3. The DOS data of (a) bare, (b) H-C-H, (c) H-C-Pd and (d) Pd-C-Pd 11-AGNR structures. Red line represents the Fermi level. Some Q0D, Q1D and 1D peaks are shown with arrows. (For interpretation of the references to color in this figure legend, the reader is referred to the web version of this article.) 


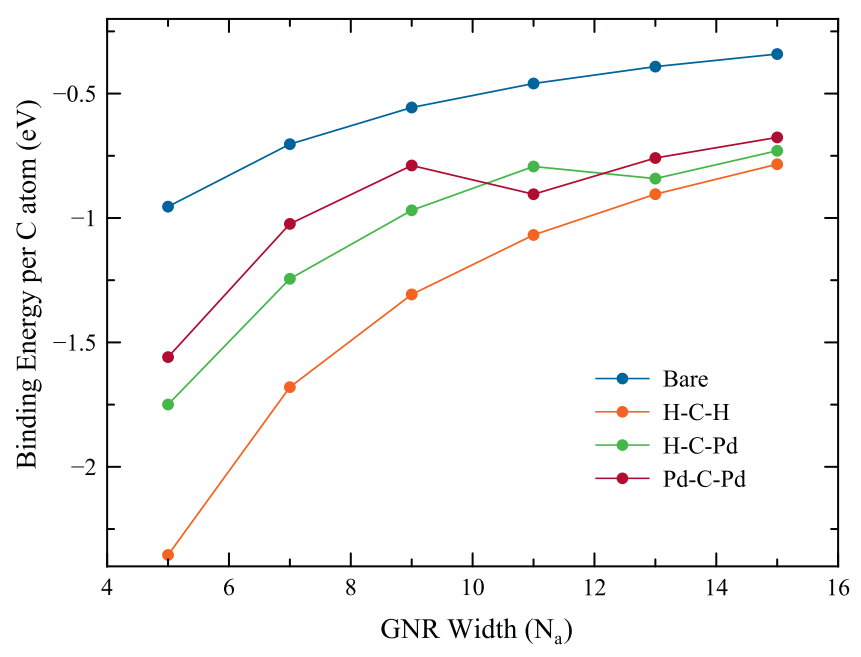

Fig. 4. The Binding energies per $C$ atom for the investigated structures.

Table 1

Total energies of the bare and the other configurations.

\begin{tabular}{rllll}
\hline \multirow{2}{*}{$N_{a}$} & \multicolumn{4}{l}{ Total energy $(\mathrm{eV})$ of configuration } \\
\cline { 2 - 5 } & Bare & H-C-H & H-C-Pd & Pd-C-Pd \\
\hline 5 & -1568.57 & -1639.98 & -3805.68 & -5975.52 \\
7 & -2200.24 & -2271.62 & -4437.27 & -6605.93 \\
9 & -2831.77 & -2903.17 & -5068.83 & -7237.33 \\
11 & -3463.32 & -3534.69 & -5700.38 & -7874.58 \\
13 & -4094.89 & -4166.26 & -6336.39 & -8505.98 \\
15 & -4726.43 & -4797.81 & -6967.94 & -9138.07 \\
\hline
\end{tabular}

number of hydrogen atoms, and number of palladium atoms, respectively. Isolated total energies are found to be $E_{P d}=$ $-1097.83 \mathrm{eV}, E_{C}=-147.31 \mathrm{eV}$ and $E_{H}=-11.97 \mathrm{eV}$. Total energies of the bare and the other configurations are listed in Table 1.

From Fig. 4, $\mathrm{H}-\mathrm{C}-\mathrm{H}$ structures have the lowest energies, which correspond with a stronger binding of the atoms. It is known that the binding energy is strongest when the $d$ shell is nearly empty or half-filled [36]. With a filled d shell, Pd has intermediate binding energy values with respect to other $4 \mathrm{~d}$ and $5 \mathrm{~d}$ transition metals
[36]. For the AGNRs with single or both side Pd termination, binding energies show a drop (actually it is an increment in magnitude that results in stronger binding) at $N_{a}=11$ and $N_{a}=13$, respectively. This situation results in a special case at $N_{a}=11$, where Pd-C-Pd structures have greater binding energies than $\mathrm{H}-\mathrm{C}-\mathrm{Pd}$ structures. With these drops, binding energy values are become similar to $\mathrm{H}-\mathrm{C}-\mathrm{H}$ structures. Therefore, one can say that for the narrower AGNRs, the Pd concentration exceeds a critical point where the binding energy shows a characteristic change. It is known that the binding energies of the GNRs are linearly dependent on Young's modulus and so the stiffness of these AGNRs [37].

In Table 2, calculated C-C, C-H $\left(d_{H}\right)$ and C-Pd $\left(d_{P d}\right)$ bond lengths for all investigated structures are given with related references $[38-41,21,42,43]$. For $C-C$ bonds, both edge $\left(d_{E}\right)$ and center $\left(d_{C}\right)$ bonds are listed in the table. For bare, $\mathrm{H}-\mathrm{C}-\mathrm{H}$ and $\mathrm{H}-\mathrm{C}-\mathrm{Pd}$ structures, center $\mathrm{C}-\mathrm{C}$ bond lengths show small variations near $1.42 \AA$. However, for Pd-C-Pd structures, center C-C bonds show decrease from $1.44 \AA$ to $1.42 \AA$ with the increasing $N_{a}$. Edge $\mathrm{C}-\mathrm{C}$ bonds show small variations for all types. $\mathrm{C}-\mathrm{H}$ bonds are in a full agreement with the literature values $[39,41]$. Bond lengths of C-Pd drop from 2.06 to $1.98 \AA$ with the increasing $N_{a}$. The value of C-Pd bonds show similarities with the Pd decorated graphene studies, where Pd decoration can be used for possible hydrogen storage [21]. The sudden difference in C-Pd bond length with the increasing $N_{a}$ is also in consistency with the drops in binding energies in this study.

Therefore, for the H-C-Pd and Pd-C-Pd structures, $N_{a}=13$ and $N_{a}=11$ can be expected as characteristic points where the stiffness of these AGNRs will be changed, respectively. Also, these characteristic points may result different hydrogen bonding of Pd atoms in Pd-decorated graphene structures, which is beyond the scope of this study.

\section{Conclusion}

In summary, we performed $a b$ initio calculations in order to study the electronic structure and binding energies of armchair graphene nanoribbons with Pd atom termination. It was found that Pd-termination can significantly influence the electronic properties of AGNRs, and induce metallicity. In DOS, many Q0D and Q1D type states are found to be induced in addition to dominant 1D states.

Table 2

Calculated lengths for C-C, C-H and C-Pd bonds for different configurations.

\begin{tabular}{|c|c|c|c|c|c|c|c|c|}
\hline \multirow{3}{*}{$\begin{array}{l}\text { Width } \\
\left(N_{a}\right)\end{array}$} & \multicolumn{8}{|c|}{ Bond lengths of different edge configurations (in Angstroms) } \\
\hline & \multicolumn{4}{|c|}{ C-C bond at near center $\left(d_{C}\right)$ and edge $\left(d_{E}\right)$ of the armchair ribbon } & \multicolumn{2}{|c|}{ C-H bond $\left(d_{H}\right)$} & \multicolumn{2}{|c|}{ C-Pd bond $\left(d_{P d}\right)$} \\
\hline & Bare & $\begin{array}{l}\mathrm{H}- \\
\text { terminated }\end{array}$ & $\begin{array}{l}\text { One edge Pd- } \\
\text { terminated }\end{array}$ & $\begin{array}{l}\text { Both edge Pd- } \\
\text { terminated }\end{array}$ & $\begin{array}{l}\mathrm{H}- \\
\text { terminated }\end{array}$ & $\begin{array}{l}\text { One edge Pd- } \\
\text { terminated }\end{array}$ & $\begin{array}{l}\text { One edge Pd- } \\
\text { terminated }\end{array}$ & $\begin{array}{l}\text { Both edge Pd- } \\
\text { terminated }\end{array}$ \\
\hline \multirow[t]{2}{*}{5} & 1.432 & 1.420 & 1.418 & 1.443 & 1.100 & 1.100 & 2.055 & 2.020 \\
\hline & 1.397 & 1.410 & 1.407 & 1.403 & & & & \\
\hline \multirow[t]{2}{*}{7} & 1.422 & 1.424 & 1.421 & 1.432 & 1.100 & 1.100 & 2.055 & 2.031 \\
\hline & 1.393 & 1.409 & 1.408 & 1.408 & & & & \\
\hline \multirow[t]{2}{*}{9} & 1.416 & 1.414 & 1.416 & 1.427 & 1.100 & 1.100 & 2.056 & 2.033 \\
\hline & 1.390 & 1.405 & 1.406 & 1.407 & & & & \\
\hline \multirow[t]{2}{*}{11} & 1.418 & 1.418 & 1.419 & 1.430 & 1.100 & 1.100 & 2.057 & 1.980 \\
\hline & 1.393 & 1.408 & 1.406 & 1.409 & & & & \\
\hline \multirow[t]{2}{*}{13} & $\begin{array}{l}1.420(1.422 \\
[7])\end{array}$ & 1.420 & 1.419 & 1.430 & 1.100 & 1.100 & 1.982 & 1.981 \\
\hline & 1.393 & 1.408 & 1.407 & 1.409 & & & & \\
\hline \multirow[t]{2}{*}{15} & 1.417 & 1.421 & 1.417 & 1.418 & 1.100 & 1.100 & 1.982 & 1.982 \\
\hline & 1.392 & 1.406 & 1.406 & 1.405 & & & & \\
\hline \multirow[t]{3}{*}{ Refs. } & $1.390[38]$ & & & & 1.090 [39] & & $2.180^{\mathrm{a}}[21]$ & \\
\hline & 1.370 [39] & & & & $1.100[41]$ & & $2.100^{\mathrm{a}}[42]$ & \\
\hline & $1.388[40]$ & & & & & & $2.140^{\mathrm{a}}[43]$ & \\
\hline
\end{tabular}

\footnotetext{
a These calculations are not edge terminated C-Pd bond length values. Values are for top sited Pd on a graphene sheet.
} 
BE and C-Pd bond lengths for H-C-Pd and Pd-C-Pd structures represent characteristic drops with the increasing GNR width. These width dependent characteristic drops of the BE and C-Pd bond lengths for the investigated GNRs may also provide information on a possible stiffness information due to the linear relation between BE and Young's modulus of GNRs. These results are important figures of merit in order to experimentally investigate in any application like hydrogen storage as to where controlled binding to graphene is required.

\section{Acknowledgements}

This work is supported by the State Planning Organization of Turkey under Grant No. 2011 K120290, by the projects DPT-HAMIT, ESF-EPIGRAT, EU-N4E, and NATO-SET-181, Gazi University BAP 05/ 2012-14, and TUBITAK under Project Nos. 107A004, 107A012, and 109E301. One of the authors (E.O.) also acknowledges partial support from the Turkish Academy of Sciences.

\section{References}

[1] K.S. Novoselov, A.K. Geim, S.V. Morozov, D. Jiang, Y. Zhang, S.V. Dubonos, I.V. Grigorieva, A.A. Firsov, Science 306 (2004) 666.

[2] C.L. Kane, E.J. Mele, Phys. Rev. Lett. 95 (2005) 226801.

[3] N.M.R. Peres, F. Guinea, A.H.C. Neto, Phys. Rev. B 72 (2005) 174406.

[4] N. Mounet, N. Marzari, Phys. Rev. B 71 (2005) 205214.

[5] M.Y. Han, B. Ozyilmaz, Y. Zhang, P. Kim, Phys. Rev. Lett. 98 (2007) 206805.

[6] A. Srivastava, A. Jain, R. Kurchania, N. Tyagi, J. Comput. Theor. Nanosci. 9 (2012) 1008

[7] Y.-W. Son, M.L. Cohen, S.G. Louie, Phys. Rev. Lett. 97 (2006) 216803.

[8] H. Zheng, Z.F. Wang, T. Luo, Q.W. Shi, J. Chen, Phys. Rev. B 75 (2007) 165414.

[9] Y.W. Son, M.L. Cohen, S.G. Louie, Phys. Rev. Lett. 97 (2006) 216803-1.

[10] Y. Kobayashi, K.I. Fukui, T. Enoki, K. Kusakabe, Phys. Rev. B 73 (2006) 1254151.

[11] L.R. Radovic, Bradley Bockrath, J. Am. Chem. Soc. 127 (2005) 5917.

[12] C. Tao, L. Jiao, O.V. Yazyev, Y.-C. Chen, J. Feng, X. Zhang, R.B. Capaz, J.M. Tour, A Zettl, S.G. Louie, H. Dai, M.F. Crommie, Nat. Phys. 7 (2011) 616.
[13] P.W. Sutter, J.-I. Flege, E.A. Sutter, Nat. Mater. 7 (2008) 406

[14] J. Coraux, A.T. N'Diaye, C. Busse, T. Michely, Nano Lett. 8 (2008) 565.

[15] P.W. Sutter, J.T. Sadowski, E.A. Sutter, Phys. Rev. B 80 (2009) 245411

[16] X. Li, W. Cai, J. An, S. Kim, J. Nah, D. Yang, R. Piner, A. Velamakanni, I. Jung, E. Tutuc, S.K. Banarjee, L. Colombo, R.S. Ruoff, Science 324 (2009) 1312.

[17] M. Xu, D. Fujita, K. Sagisaka, E. Watanabe, N. Hanagata, ACS Nano 5 (2011) 1522.

[18] S.-Y. Kwon, C.V. Ciobanu, V. Petrova, V.B. Shenoy, J. Bareno, V. Gambin, I. Petrov, S. Kodambaka, Nano Lett. 9 (2009) 3985.

[19] Y. Murata, E. Starodub, B.B. Kappes, C.V. Ciobanu, N.C. Bartelt, K.F. McCarty, S Kodambaka, Appl. Phys. Lett. 97 (2010) 143114.

[20] J.-H. Gao, N. Ishida, I. Scott, D. Fujita, Carbon 50 (2012) 1674

[21] I. Lopez-Corral, E. German, A. Juan, M.A. Volpe, G.P. Brizuela, J. Phys. Chem. C 115 (2011) 4315.

[22] V.B. Parambhath, R. Nagar, S. Ramaprabhu, Langmuir 28 (2012) 7826.

[23] R. Thapa, D. Sen, M.K. Mitra, K.K. Chattopadhyay, Phys. B: Condens. Matter 406 (2011) 368 .

[24] I. Cabria, M.J. López, J.A. Alonso, Phys. Rev. B 81 (2010) 035403.

[25] Version 12.2 QuantumWise A/S <http://www.quantumwise.com>.

[26] M. Brandbyge, J.-L. Mozos, P. Ordejón, J. Taylor, K. Stokbro, Phys. Rev. B 65 (2002) 165401.

[27] J.M. Soler, E. Artacho, J.D. Gale, A. García, J. Junquera, P. Ordejón, D. SánchezPortal, J. Phys.: Condens. Matter 14 (2002) 2745

[28] J.P. Perdew, A. Zunger, Phys. Rev. B 23 (1981) 5048.

[29] J. Zhao, A. Buldum, J. Han, J.P. Lu, Nanotechnology 13 (2002) 195.

[30] L. Yang, M. Cohen, S. Louie, Nano Lett. 7 (2007) 3112.

[31] H.J. Monkhorst, J.D. Pack, Phys. Rev. B 13 (1976) 5188.

[32] N.K. Jaiswal, P. Srivastava, Physica E 44 (2011) 75.

[33] H. Xiang, E. Kan, S.-H. Wei, M.-H. Whangbo, J. Yang, Nano Lett. 9 (2009) 4025.

[34] Y. Li, Z. Zhou, P. Shen, Z. Chen, J. Phys. Chem. C 113 (2009) 15043.

[35] H. Cho, P.R. Prucnal, J. Vac. Sci. Technol. B 7 (1989) 1363.

[36] V. Zolyomi, A. Rusznyak, J. Kurti, C.J. Lambert, J. Phys. Chem. C 114 (2010) 18548.

[37] C.D. Zeinalipour-Yazdi, C. Christofides, J. Appl. Phys. 106 (2009) 054318.

[38] P. Koskinen, S. Malola, H. Hakkinen, Phys. Rev. Lett. 101 (2008) 115502.

[39] N. Gorjizadeh, A.A. Farajian, K. Esfarjani, Y. Kawazoe, Phys. Rev. B 78 (2008) 155427.

[40] C. Jin, H. Lan, L. Peng, K. Suenaga, S. Iijima, Phys. Rev. Lett. 102 (2009) 205501

[41] E.J. Duplock, M. Scheffler, P.J.D. Lindan, Phys. Rev. Lett. 92 (2004) 225502-1.

[42] K.T. Chan, J.B. Neaton, M.L. Cohen, Phys. Rev. B 77 (2008) 235430.

[43] I. Cabria, M.J. Lopez, J.A. Alonso, Phys. Rev. B 81 (2010) 035403. 